\title{
ARC syndrome: an expanding range of phenotypes
}

K M Eastham, P J McKiernan, D V Milford, P Ramani, J Wyllie, W van't Hoff, S A Lynch, A A M Morris

Department of

Paediatrics, James Cook University Hospital, Marton

Road, Middlesbrough, Cleveland TS4 3BW, UK

K M Eastham

Department of Neonatology, James Cook University Hospital J Wyllie

Liver Unit, Children's Hospital NHS Trust, Birmingham $\mathrm{B} 4$ 6NH, UK

P J McKiernan

Department of Nephrology, Children's Hospital NHS Trust,

Birmingham

D V Milford

Department of

Histopathology,

Children's Hospital

NHS Trust,

Birmingham

P Ramani

Nephro-Urology Unit, Institute of Child

Health, University

College London

Medical School,

London, UK

W van't Hoff

Department of Human

Genetics, 19

Claremont Place,

Newcastle upon Tyne

NE2 4AA, UK

$S$ A Lynch

Department of Metabolic Medicine, Great Ormond Street

Hospital, London

WC1N 3JH, UK

A A M Morris

Correspondence to:

Dr Morris

morria1@gosh.nhs.uk

Accepted 20 August 2001
Abstract

Aim-To describe the clinical phenotype in infants with ARC syndrome, the association of arthrogryposis, renal tubular acidosis, and cholestasis.

Methods-The medical records for six patients with ARC syndrome were reviewed, presenting over 10 years to three paediatric referral centres.

Results-All patients had the typical pattern of arthrogryposis. Renal Fanconi syndrome was present in all but one patient, who presented with nephrogenic diabetes insipidus. Although all patients had severe cholestasis, serum $\gamma$ glutamyltransferase values were normal. Many of our patients showed dysmorphic features or ichthyosis. All had recurrent febrile illnesses, diarrhoea, and failed to thrive. Blood films revealed abnormally large platelets.

Conclusions-ARC syndrome exhibits notable clinical variability and may not be as rare as previously thought. The association of Fanconi syndrome, ichthyosis, dysmorphism, jaundice, and diarrhoea has previously been reported as a separate syndrome: our observations indicate that it is part of the ARC spectrum. (Arch Dis Child 2001;85:415-420)

Keywords: arthrogryposis; renal tubular acidosis; Fanconi syndrome, cholestasis

ARC syndrome refers to an association between arthrogryposis, renal tubular dysfunction, and cholestasis. Eleven pedigrees have been reported since the association was first described in 1973. ${ }^{1-8}$ Autosomal recessive inheritance is suggested by the frequency of parental consanguinity and recurrence in siblings.

As more patients with ARC syndrome have been identified, it has become apparent that there is notable clinical variability, even within the same family. Affected siblings with and without arthrogryposis have been reported. ${ }^{8}$ Renal tubular dysfunction ranges from isolated renal tubular acidosis to complete Fanconi syndrome, and hepatic histology shows variable combinations of cholestasis, intrahepatic biliary hypoplasia, giant cell hepatitis, lipofuscin deposition, and fibrosis, ultimately progressing to cirrhosis. Additional features have been reported in some patients, including failure to thrive, nephrogenic diabetes insipidus, neurogenic muscular atrophy (which appears to be responsible for the arthrogryposis), cerebral malformations, and nerve deafness. ${ }^{8}$ Most patients die by the age of 7 months, but those surviving longer have shown severe developmental delay. ${ }^{8}$

We report a further six patients, from five pedigrees. Additional clinical features in our patients included dysmorphism, ichthyosis, diarrhoea, recurrent febrile illnesses, and abnormal platelets. Similar features have been reported by Deal et al in patients from four consanguineous families ${ }^{9}$; we suspect that these patients also had ARC syndrome.

\section{Methods}

We have reviewed all cases of ARC syndrome referred to three paediatric centres in the UK, over a 10 year period from April 1990. Clinical features and results of investigations were obtained from the medical records, and histology was reviewed.

\section{Case reports}

PATIENT 1

This girl was the first child of healthy, first cousin, Pakistani parents. There is a complex family history of intermarriage and three cousins died as neonates of unknown aetiology. Pregnancy was complicated by oligohydramnios. The baby had a birth weight of $3.23 \mathrm{~kg}$, and was noted to have a sloping forehead, small anterior fontanelle, a high arched palate, a low set posteriorly rotated left ear, and redundant skin folds in the neck. There was generalised hypotonia and multiple contractures, including talipes calcaneovalgus, flexion contractures of the knees, dislocated hips, radial deviation of the wrists, and camptodactyly of the fingers (fig 1). Muscle bulk of deltoid and triceps was notably reduced. Within 72 hours, she developed renal tubular acidosis and cholestatic jaundice. Diarrhoea, recurrent sepsis, and failure to thrive ensued despite treatment with sodium bicarbonate, phenobarbitone, vitamin $\mathrm{K}$, and a formula milk high in medium chain triglycerides. The baby died of pneumonia at 11 weeks of age. Permission for autopsy was refused.

\section{PATIENT 2}

This female sibling of case 1 was born at term with a birth weight of $2.99 \mathrm{~kg}$. Antenatal scans had shown oligohydramnios, reduced fetal movements, and arthrogryposis. At birth, she was noted to have upward slanting eyes, an alar appearance to the mouth, a high arched palate, and redundant posterior skin folds in the neck. The baby was hypotonic with contractures similar to her sister. She developed hyperchloraemic metabolic acidosis and jaundice on day 3 and was treated symptomatically. She died at home at the age of 5 days. A liver biopsy specimen was obtained postmortem. 


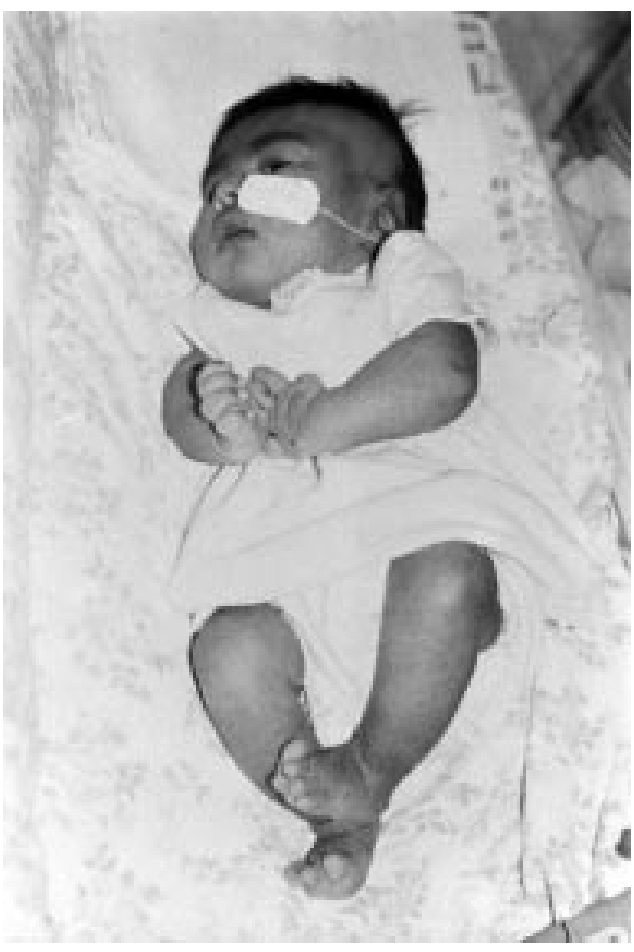

Figure 1 Infant with ARC syndrome.

PATIENT 3

This boy was the third child of first cousin Pakistani parents. His two siblings are healthy. Antenatal scans showed hydronephrosis and bilateral talipes equinovarus. He was delivered by caesarean section for a footling breech presentation, with a birth weight of $3.37 \mathrm{~kg}$. Bilateral femoral fractures were identified following delivery. He had a beaked nose, small anterior fontanelle, lax skin, low set thumbs, and cryptorchidism. Multiple contractures of the lower limbs were present, including talipes equinovarus, flexion contractures of the knees, and dislocated hips. A skeletal survey showed slender long bones and ribs. He developed cholestatic jaundice and renal tubular defects, including acidosis, by 10 days of age and was treated with sodium bicarbonate, phosphate, and potassium chloride supplementation. Subsequently, he had diarrhoea, recurrent fever, and failed to thrive. Despite normal coagulation studies and platelet numbers, a liver biopsy at seven weeks led to a haemoperitoneum, which required laparotomy for evacuation. $\mathrm{He}$ died at 3 months of age.

PATIENT 4

This male infant was born at term to non-consanguineous Pakistani parents, with a birth weight of $3.65 \mathrm{~kg}$. There are five healthy siblings. He was noted to be hypotonic with right talipes equinovarus and mild flexion contractures of all limbs. He developed renal tubular acidosis and conjugated hyperbilirubinaemia by the age of 4 days. He failed to thrive despite treatment with bicarbonate, fat soluble vitamins, and a medium chain triglyceride based feed. Cranial computed tomography revealed a large porencephalic cyst in the left cerebral hemisphere. The clinical course was complicated by recurrent infections, including bronchiolitis caused by respiratory syncytial virus, and acute on chronic renal failure. $\mathrm{He}$ died at 5 months of age.

\section{PATIENT 5}

This girl, the second child of healthy first cousin Pakistani parents, was born at term with a birth weight of $2.84 \mathrm{~kg}$. Reduced fetal movement had been noted from 28 weeks gestation; multiple contractures were noted at birth, including bilateral talipes equinovarus and flexion contractures of the knees and hips. Within 24 hours, she became hypoglycaemic and hypothermic. At 12 days of age, she was noted to be hypotonic with cholestatic jaundice, diarrhoea, poor feeding, and weight loss (11\% birth weight). Recurrent febrile illnesses ensued and she failed to thrive, despite receiving $0.92 \mathrm{~mJ} / \mathrm{kg} / \mathrm{day}$. At the age of 2 months she developed polyuria and renal tubular acidosis. A renal biopsy was undertaken at 3 months of age, following which she bled heavily, despite normal clotting studies and platelet numbers. This led to a cardiac arrest from which she could not be resuscitated. A younger sister has recently been born with similar features, and a maternal cousin has also had two children with ARC syndrome.

\section{PATIENT 6}

This female infant was born by elective caesarean section, for breech presentation, with a birth weight of $2.94 \mathrm{~kg}$. The parents are first cousins of Pakistani origin, and have one other healthy child. The baby was noted to have lax skin at the neck, radial deviation of the wrists, talipes calcaneovalgus, and dislocated hips. She had an undisplaced supracondylar fracture of the right femur following delivery. She was admitted at 18 days of age with poor feeding and cholestatic jaundice. Stools were loose and pale in colour. Blood thyroid stimulating hormone was $65 \mathrm{mU} / 1$ (normal 0.4-3.5 mU/1) with a free thyroxine of $12.4 \mathrm{pmol} / 1$ (normal 13.8-22.5 pmol/1). Transient hypothyroidism was suspected and she was treated with thyroxine in addition to fat soluble vitamins, alpha calcidol, and phenobarbitone. At 4 weeks of age, she was noted to produce large quantities of dilute urine, with evidence of nephrogenic diabetes insipidus. She failed to thrive, developed cholestatic liver disease, and died at age 4-5 months.

\section{Results}

\section{BLOOD FILMS}

Blood films were available for patients 3, 4, 5, and 6 . In all cases these showed abnormally large platelets, with a measured mean platelet diameter, for patient 3, of $10 \mu \mathrm{m}$ (normal 3 $\mu \mathrm{m})$. Formal testing of platelet function was not undertaken.

\section{RENAL INVESTIGATIONS}

Table 1 summarises standard tests of renal tubular function. Urinary protein:creatinine ratios, measured in patients $3,4,5$, and 6 , were raised in all $(1355-4000 \mathrm{mg} / \mathrm{mmol}$, normal <20). Patients 1, 3, 4, and 5 had episodes of 
Table 1 Renal and hepatic investigations

\begin{tabular}{|c|c|c|c|c|c|c|c|}
\hline Patient & 1 & 2 & 3 & 4 & 5 & 6 & $\begin{array}{l}\text { Normal } \\
\text { values }\end{array}$ \\
\hline Blood pH & 7.31 & 7.25 & 7.34 & 7.31 & 7.25 & 7.36 & $7.35-7.45$ \\
\hline Bicarbonate (mmol/1) & 14.3 & 16.8 & 17.2 & 16 & 16 & 21.5 & $22-29$ \\
\hline Anion gap $(\mathrm{mmol} / \mathrm{l})$ & 16 & 15 & 16 & 17 & 15 & NK & $15-20$ \\
\hline Urine $\mathrm{pH}$ & 6.4 & 6.4 & 6.5 & 6.0 & 6.0 & 5.6 & \\
\hline TRP (\%) & NK & NK & 42 & 68 & 59.8 & 74.1 & $80-100$ \\
\hline Aminoaciduria & + & + & + & NK & + & - & \\
\hline Glycosuria & + & + & + & NK & - & - & \\
\hline Creatinine $(\mu \mathrm{mol} / \mathrm{l})$ & 76 & 62 & 71 & 96 & 54 & 69 & $33-85$ \\
\hline \multicolumn{8}{|l|}{ Bilirubin $(\mu \mathrm{mol} / \mathrm{l})$} \\
\hline Total & 287 & $95^{\star}$ & 252 & 234 & 118 & 162 & \\
\hline Conjugated & 183 & 31 & 204 & 88 & 95 & 154 & \\
\hline Alkaline phosphatase $(\mathrm{U} / \mathrm{l}) \dagger$ & 832 & 239 & 1042 & 1475 & 2258 & 1652 & $60-350$ \\
\hline $\operatorname{AST}(\mathrm{U} / \mathrm{l})$ & NK & 27 & 22 & 328 & 68 & 42 & $1-45$ \\
\hline $\operatorname{ALT}(\mathrm{U} / \mathrm{l})$ & 24 & NK & 25 & 273 & 44 & 22 & $3-50$ \\
\hline$\gamma \mathrm{GT}(\mathrm{U} / \mathrm{l})$ & NK & 24 & 33 & NK & 58 & 32 & $1-65$ \\
\hline
\end{tabular}

$\star$ No measurements were made beyond age 3 days.

tSerum phosphate concentrations were normal at the time of the raised alkaline phosphatase values in all patients.

TRP, tubular reabsorption of phosphate; NK, not known; +, present; -, absent; AST, aspartate transaminase; ALT, alanine transaminase; $\gamma \mathrm{GT}, \gamma$ glutamyltransferase.

hypernatraemic dehydration but nephrogenic diabetes insipidus was proved only in patient 6 (maximum urinary osmolality after desmopressin acetate $250 \mathrm{mOsm} / \mathrm{kg}$ ). Ultrasound scans showed small dysplastic kidneys in patients 1 and 2. In patients 3 and 4, there was loss of corticomedullary differentiation and the medulla appeared echobright, consistent with nephrocalcinosis.

Renal histology was available for patients 4 and 5. Both biopsy specimens showed acute and chronic inflammation of the interstitium with focal multinucleate giant cell reaction to the contents of an occasional ruptured tubule. Tubular calcification was not evident. Electron microscopic examination did not reveal any significant abnormality. Patient 4 showed features of multicystic dysplasia, comprising a bar of cartilage with a few ducts and tubules surrounded by concentric collarettes of immature mesenchyme. There was cystic dilatation of many of the tubules. The glomeruli were fetal in configuration and $10 \%$ showed focal segmental and global sclerosis. The tufts were retracted with expansion of the Bowman's space and occasional glomerular cysts. Renal histology for patient 5 showed good preservation of tubules, without cystic dilatation. A few glomeruli showed expansion of the Bowman's spaces, but no cysts were evident. There was no glomerular sclerosis. Immunofluorescence for immunoglobulins and complement components was negative.

\section{LIVER INVESTIGATIONS}

Table 1 summarises relevant biochemical investigations. Ultrasound examination in patients $2-5$ showed normal liver texture with no biliary dilatation. Technetium labelled methyl bromoiminodiacetic acid scans of biliary excretion were performed in patients $1,4,5$, and 6 . In all cases, there was good uptake of isotope. For patient 1, no isotope appeared in the gall bladder or bowel after 48 hours, but patients 4 and 6 showed normal excretion. Patient 5 showed no excretion into the bowel at 5 weeks, but this was shown on a repeat scan at 8 weeks of age.
Table 2 Liver histology

\begin{tabular}{lllll}
\hline Patient & 2 & 3 & 4 & 5 \\
\hline Giant cell transformation & + & + & + & + \\
Paucity of bile ducts & - & + & - & - \\
Bile plugs or intracellular pigment & + & + & + & + \\
Bile ductule proliferation & + & - & + & - \\
Lipofuscin & - & - & + & + \\
Portal tract fibrosis & - & - & + & - \\
\hline
\end{tabular}

The liver biopsy specimens were examined using haematoxylin and eosin, Masson trichrome, Elastin Van-Gieson, Reticulin, Periodic Acid-Schiff with and without diastase digestion, Perls, Orcein, Schmorl's, and long Ziehl-Neelson stains. The renal biopsy specimens were examined at multiple levels using haematoxylin and eosin, Martius scarlet blue, silver methanamine stain, and Periodic AcidSchiff. Immunofluorescence studies were performed on patient 5 . Liver and renal biopsy specimens were examined by electron microscopy. Table 2 summarises liver histology.

\section{NEUROMUSCULAR INVESTIGATIONS}

Electromyography in patient 3 showed neurogenic changes with reduced compound muscle action potentials. In patient 5, electromyography was normal but a muscle biopsy sample showed atrophy with variable sized muscle fibres, compatible with denervation. Neurogenic muscle atrophy was also seen in a muscle biopsy sample from patient 4 .

\section{ADDITIONAL INVESTIGATIONS}

Blood immunoreactive trypsin, measured in patients 4 and 6 , was raised in both $(>1000$ and $358 \mu \mathrm{g} / \mathrm{l}$, respectively; normal <120). Sweat chloride was not measured. In each pedigree, investigations excluded chromosomal abnormalities, congenital infections, peroxisomal biogenesis disorders, $\alpha_{1}$ antitrypsin deficiency, galactosaemia, organic acidaemias, and amino acidopathies. All patients had normal blood lactate concentrations; normal CSF lactate concentrations were shown in patients 3 and 5 . For patients 4 and 5, studies of the mitochondrial respiratory chain were performed on muscle, with normal results.

\section{Discussion}

ARC syndrome is a rare autosomal recessive condition. Over recent years it has become apparent that the phenotype is variable; cases may go undiagnosed as not all patients present with the three cardinal features. ${ }^{145}$ It has also been recognised that the condition encompasses syndromes originally thought to be distinct. Initially, patients were classified according to their hepatic histology into two groups, characterised either by intrahepatic biliary hypoplasia and giant cell hepatitis or cholestasis with lipofuscin deposition. In 1994, Horslen et al found all the histological abnormalities in a single patient and concluded that both groups of patients had the same condition. ${ }^{2}$

Our six cases all had arthrogryposis, renal tubular dysfunction, and cholestatic liver disease. The arthrogryposis is thought to result from neurogenic muscle atrophy. Consistent 
Table 3 Features in patients from the current series and those reported by Deal et al

\begin{tabular}{|c|c|c|c|c|c|c|c|c|c|}
\hline \multirow{2}{*}{$\begin{array}{l}\text { Reference } \\
\text { Pedigree }\end{array}$} & \multicolumn{5}{|c|}{ Current series } & \multicolumn{4}{|l|}{ Deal et al } \\
\hline & 1 & 2 & 3 & 4 & 5 & 1 & 2 & 3 & 4 \\
\hline Affected cases $\dagger$ & $2(1,2)$ & $1(3)$ & $1(4)$ & $1(5)$ & $1(6)$ & 3 & 2 & 1 & 2 \\
\hline Ethnic origin & Pakistan & Pakistan & Pakistan & Pakistan & Pakistan & Pakistan & Pakistan & Pakistan & Oman \\
\hline Consanguineous & + & + & - & + & + & + & + & + & + \\
\hline Arthrogryposis & + & + & + & + & + & + & + & + & + \\
\hline Neurogenic muscle change & & + & + & + & & + & + & & \\
\hline Fanconi syndrome & + & + & + & + & - & + & + & + & + \\
\hline Nephrogenic diabetes insipidus & $\star$ & $\star$ & $\star$ & $\star$ & + & $\star$ & $\star$ & $\star$ & \\
\hline Cholestasis & + & + & + & + & + & + & + & + & + \\
\hline Lax skin & + & + & & & + & & & & \\
\hline Ichthyosis & & & & + & & + & + & + & + \\
\hline Sepsis/fevers & + & + & + & + & + & + & + & + & + \\
\hline Diarrhoea & + & + & + & + & + & + & + & + & + \\
\hline Failure to thrive & + & + & + & + & + & + & + & + & + \\
\hline Abnormal platelets & & + & + & + & + & + & & & + \\
\hline
\end{tabular}

${ }^{\star}$ Episodes of hypernatraemic dehydration but nephrogenic diabetes insipidus not formally shown.

†Numbers in parentheses refer to case numbers in current series.

+, present; -, absent; blank, not mentioned.

with this, all our patients were hypotonic, and in three, evidence of denervation was provided by muscle histology or electromyography.

Most reported patients with ARC syndrome have had multiple features of Fanconi syndrome, including aminoaciduria, glycosuria, phosphaturia, and bicarbonate wasting. Renal tubular acidosis has generally been the most striking clinical abnormality, but many patients have also had features to suggest nephrogenic diabetes insipidus. Our patients all had renal tubular acidosis and other features of Fanconi syndrome, with the exception of patient 6 , in whom the principal renal tubular abnormality was nephrogenic diabetes insipidus (table 1). Patients 1, 3, 4, and 5 also produced large quantities of dilute urine and had recurrent episodes of hypernatraemic dehydration, although formal testing for nephrogenic diabetes insipidus was not undertaken. Renal histology is variable. Nephrocalcinosis has been noted in several previous patients, ${ }^{13578}$ but it was not evident in either of our patients who underwent renal biopsies. Multicystic dysplasia was the main histological feature for patient 4 , and in patients 1 and 2, the ultrasound appearances also suggested dysplasia. Renal histology for patient 5 was compatible with interstitial nephritis.

Liver histology in our patients differed from the usual pattern in a few minor respects. All showed cholestasis with multinucleate giant cell transformation of hepatocytes. Intrahepatic biliary hypoplasia and lipofuscin deposition were less consistent. Indeed, patient 2 showed neither of these abnormalities. Previous cases have consistently shown at least one of these changes. ${ }^{1-8}$ We do not consider the absence of these changes to be significant. The number of intrahepatic bile ducts, for example, depends on timing and site of the biopsy. Bile ducts develop from the hilar region to the periphery of the liver; the intrahepatic bile duct system is immature at birth, and does not have an adult appearance until one month post term. ${ }^{10}$ Histopathology, therefore, depends on the timing and site of the biopsy.

In addition to the three cardinal features of ARC syndrome, our patients showed a number of other features (table 3), most of which have been described previously (table 4). Failure to thrive has been a problem in all patients. Diarrhoea is common, ${ }^{25}$ and is probably secondary to fat malabsorption. Presumably, this and the renal tubular losses both contribute to the patients' universal failure to thrive. All our patients and several previous patients have had recurrent febrile illnesses. ${ }^{35}$ Patient 5 in our series had ichthyosis, as did patients from four previous pedigrees. ${ }^{378}$ Dysmorphic features seen in our patients included lax skin (particularly in the neck), low set ears, high arched palate, proximally placed thumbs, and cryptorchidism; all, except cryptorchidism, have been noted previously. ${ }^{25} 78$

Table 4 Features of published cases of ARC syndrome

\begin{tabular}{|c|c|c|c|c|c|c|c|c|c|c|c|}
\hline \multirow{2}{*}{$\begin{array}{l}\text { Reference } \\
\text { Pedigree }\end{array}$} & \multirow{2}{*}{$\begin{array}{l}1 \\
1\end{array}$} & \multicolumn{2}{|l|}{2} & \multicolumn{3}{|l|}{3,4} & \multirow{2}{*}{$\begin{array}{l}5 \\
1\end{array}$} & \multirow{2}{*}{$\begin{array}{l}6 \\
1\end{array}$} & \multirow{2}{*}{$\begin{array}{l}7 \\
1\end{array}$} & \multicolumn{2}{|l|}{8} \\
\hline & & 1 & 2 & 1 & 2 & 3 & & & & 1 & 2 \\
\hline Affected cases & 2 & 1 & 2 & 2 & 1 & 2 & 4 & 4 & 2 & 2 & 2 \\
\hline Ethnic origin & Swiss & Pakistan & Asian & Italian & Italian & Italian & $\begin{array}{l}\text { North } \\
\text { African }\end{array}$ & Portugal & Lebanon & & \\
\hline Consanguineous & + & + & + & + & - & - & - & + & + & & + \\
\hline Arthrogryposis & + & + & + & + & + & + & + & + & + & $+/-$ & $+/-$ \\
\hline $\begin{array}{l}\text { Neurogenic muscle change } \\
\text { Fanconi syndrome }\end{array}$ & & & & + & & & & + & & & \\
\hline Fanconi syndrome & + & + & + & + & + & + & + & + & + & + & + \\
\hline Nephrogenic diabetes insipidus & & + & & + & + & & + & + & & + & + \\
\hline Cholestasis & + & + & + & + & + & + & + & + & + & + & \\
\hline Lax skin & & & + & & & & & & & & \\
\hline Ichthyosis & & & & + & & & & & + & + & $+/-$ \\
\hline Sepsis/fevers & & & & & + & + & + & & & & \\
\hline Diarrhoea & & + & & & & & + & & & & \\
\hline Failure to thrive & + & + & + & + & + & + & + & + & + & + & + \\
\hline
\end{tabular}

+, present; -, absent; blank, not mentioned; +/-, present in some affected members of pedigree but not others. 
Abnormally large platelets were seen in all our patients for whom blood films were examined (patients 3, 4, 5, and 6). None of the patients had thrombocytopenia and formal studies of platelet function were not undertaken. The histories, however, suggest that the patients may have had a bleeding tendency. Thus, patients 3 and 5 suffered significant bleeds following liver and renal biopsies, respectively, despite normal clotting studies. One previous patient with ARC syndrome also died from haemorrhage following a liver biopsy, despite normal clotting studies, ${ }^{3}$ and another patient died as a result of a cerebral bleed, though clotting studies were not reported in this case. ${ }^{8}$

In 1990, Deal et al described eight patients with Fanconi syndrome, ichthyosis, dysmorphism, jaundice, and diarrhoea. ${ }^{9}$ At the time, few cases of ARC syndrome had been reported and neither the variability nor the full range of features was appreciated. Distinction was made on the basis of liver histology and haematological findings. Liver histology in the patients of Deal et al showed cholestasis and giant cell transformation of hepatocytes but no lipofuscin deposition or bile duct hypoplasia. As discussed previously, we do not now consider these features necessary for the diagnosis of ARC syndrome - neither was present in patient 2 from our series. The other distinguishing feature reported by Deal et al was an abnormality of platelet morphology. Blood films were only available in two cases. In both of these, platelets were abnormally large, lacked alpha granules, and had low concentrations of $\beta$ thromboglobulin, platelet factor 4, and thrombospondin. The features were interpreted as suggesting "grey platelet syndrome", though this is usually associated with thrombocytopenia $^{11}$ (platelet numbers were normal in these patients). As mentioned above, our patients also had normal numbers of abnormally large platelets. Other features reported by Deal et al, such as dysmorphism and ichthyosis, were also seen in our patients (table 3 ). We conclude that the patients described by Deal et al suffered from ARC syndrome.

While the pedigrees in our series and previous reports support an autosomal recessive pattern of inheritance, the genetic basis for ARC syndrome remains unknown. Most causes of Fanconi syndrome involve a metabolic abnormality that reduces the availability of ATP and thereby reduces sodium coupled solute transport. ${ }^{12}$ We and others, however, have been unable to identify a metabolic aetiology in ARC syndrome. A membrane transport defect might explain the combination of Fanconi syndrome, cholestasis, neurological dysfunction, and platelet abnormalities. In this context, it is interesting to note that serum $\gamma$ glutamyltransferase values were consistently normal in our patients (table 1). In most cholestatic conditions, $\gamma$ glutamyltransferase is released from the biliary epithelium by the detergent effect of bile acids, leading to high serum concentrations. Normal $\gamma$ glutamyltransferase values are seen in progressive familial intrahepatic cholestasis (PFIC), a group of autosomal recessive diseases characterised by progressive cholestatic liver disease with impaired bile acid transport. ${ }^{13}$ Diarrhoea is a major clinical feature of PFIC1, and was seen in all our patients. PFIC1 is caused by a mutation in a single gene, FIC $1 .{ }^{14} \mathrm{FIC} 1$ is expressed in a wide variety of tissues, including liver, brain, kidney, pancreas, and skeletal muscle, and is particularly high in the small intestine ${ }^{14}$ It is possible that different mutations in this gene may cause a more severe phenotype, as seen in patients with ARC syndrome, and we consider the gene a good candidate.

A number of candidate genes causing Fanconi syndrome have been identified in the mouse. One such gene, hepatocyte nuclear factor 1 (HNF1), is a transcriptional activator of many hepatic genes, and is predominantly expressed in the liver and kidney. ${ }^{15}$ Mice lacking HNF1 fail to thrive and die, around the time of weaning, after a progressive wasting syndrome and cholestasis. Mutant mice also suffer from Fanconi syndrome and phenylketonuria. Raised phenylalanine concentrations were not seen in our patients with ARC syndrome; however, it is known that although the mouse-man phenotype can correlate closely, there can be discrepancies. We therefore consider that HNF1 is also a good candidate gene.

All of our patients and most of those reported by Deal et al were immigrants from Pakistan. This community has a high frequency of consanguineous marriages; progress in determining the aetiology of ARC syndrome is most likely to result from identifying the responsible gene by autozygosity mapping and mutation analysis, using a candidate gene approach.

We are grateful to Prof. PT Clayton and Drs C Close, A Duthie, A Goldstein, and U Wariyar for allowing us to include their patients, and to Dr C Wright for undertaking histopathology on patient 2 .

1 Lutz-Richner AR, Landolt RF. Familiare Gallengangsmissbildungen mit tubularer Neireninsurfizienz. Helv Paediatr Acta 1973;28:1-12.

2 Horslen SP, Quarrell OWJ, Tanner MS. Liver histology in the arthrogryposis multiplex congenita, renal dysfunction and cholestasis (ARC) syndrome: report of three new cases and review. 7 Med Genet 1994;31:62-4.

3 Di Rocco M, Callea F, Pollice B, et al. Arthrogryposis, renal dysfunction and cholestasis syndrome: report of five patients from three Italian families. Eur F Pediatr 1995;154: 835-9.

4 Di Rocco M, Reboa E, Barabino A, et al. Arthrogryposis, cholestatic pigmentary liver disease and renal dysfunction: report of a second family. Am ₹ Med Genet 1990;37:23740 .

5 Nezelof C, Dupart MC, Jaubert F, Elliachar E. A lethal familial syndrome associating arthrogryposis multiplex congenita, renal dysfunction and cholestatic pigmentary liver disease. F Pediatr 1979;94:258-60.

6 Saraiva JM, Lenos C, Goncalves I, et al. Arthrogryposis multiplex congenita with renal and hepatic abnormalities in a female infant. 7 Pediatr 1990;117:761-3.

7 Mikati MA, Barakat AY, Sulh HB, Der Kaloustian VM. Renal tubular insufficiency, cholestatic jaundice and multiple congenital anomalies - a new multisystem syndrome. Helv Paediatr Acta 1984;39:463-71.

8 Coleman RA, Van Hove JLK, Morris R, et al. Cerebral defects and nephrogenic diabetes insipidus with the ARC syndrome: additional findings or a new syndrome (ARCCNDI)? Am f Med Genet 1997;72:335-8. 
9 Deal JE, Barratt M, Dillon MJ. Fanconi syndrome, icthyosis, dysmorphism, jaundice, diarrhoea-a new syndrome. Pedidysmorphism, jaundice, diar
atr Nephrol 1990;4:308-13.

10 Van Eyken P, Sciot R, Callea F, et al. The development of the intrahepatic bile ducts in man; a keratinimmunohistochemical study. Hepatology 1988;6:1586-95.

11 Raccuglia G. Gray platelet syndrome- a variety of qualitative platelet disorder. Am f Med 1971;51:818-28.

12 Foreman J. Cystinosis and Fanconi syndrome. In: Barratt TM, Avner ED, Harmon WE, eds. Pediatric nephrology, 4th edn. Philadelphia: Lippincott, Williams \& Wilkins, 1999:593-607.
13 Strautnieks SS, Bull LN, Knisley AS, et al. A gene encoding a liver-specific $\mathrm{ABC}$ transporter is mutated in progressive familial intrahepatic cholestasis. Nat Genet 1998;20:233-

14 Bull LN, van Eijk MJT, Pawlikowska L, et al. A gene encoding a P-type ATPase mutated in two forms of hereditary cholestasis. Nat Genet 1998;18:219-24.

15 Pontoglio M, Barra J, Hadchouel M, et al. Hepatocyte nuclear factor 1 inactivation results in hepatic dysfunction, phenylketonuria and renal Fanconi syndrome. Cell 1996; 84:575-85.

\section{Epilepsy and mortality}

Children with epilepsy have an increased mortality rate but the increase is largely confined to those with symptomatic epilepsy and sudden unexpected death in epilepsy (SUDEP) seems to be rare in childhood although adults with SUDEP often have epilepsy dating from childhood. A Dutch study (Petra M C Callenbach and colleagues. Pediatrics 2001;107:1259-63) has provided more data about children.

A total of 472 children aged 1 month to 16 years with newly diagnosed epilepsy were followed up for 5 years during which nine died (Mortality 3.8/1000 person years (seven times population expected rate)). None of the 328 children with nonsymptomatic epilepsy died. The nine deaths in the group of 144 children with symptomatic epilepsy gave a mortality rate of $129 / 1000$ person years (23 times expected rate). Of these nine children, three had progressive neurological disease (Niemann-Pick disease, cerebral ependymoma, infantile ceroid lipofuscinosis) and six had a static encephalopathy. None was thought to have died during a seizure and none was considered to be a case of SUDEP. Most deaths had a respiratory cause.

Children with nonsymptomatic epilepsy have a low risk of death within 5 years of epilepsy onset. Children with symptomatic epilepsy are at high risk of dying from pneumonia or other respiratory cause. SUDEP did not occur in this series.

ARCHIVIST 